Paper

\title{
Assessment of statin therapy in non-diabetic patients attending a medical clinic in a tertiary care institution of Sri Lanka
}

\author{
Jayasinghe I K ${ }^{1}$, Rajapaksha $\mathbf{B} \mathbf{S} \mathbf{J}^{1}$, De Silva K P $\mathbf{M} \mathbf{R}^{1}$, Gunawardena $\mathbf{W} \mathbf{P} \mathbf{M}^{1}$, Kumarasinghe $\mathbf{C}$ G ${ }^{1}$, \\ Mazeen S A M'1, Abeywickrama $\mathbf{U} \mathbf{K}^{2}$
}

Journal of the Ceylon College of Physicians, 2018, 49, 64-69

\begin{abstract}
Background: Dyslipidaemia is a major risk factor for Atherosclerotic Cardiovascular Disease (ASCVD) and cholesterol lowering treatment reduces cardiovascular risk. The 2013 American College of Cardiology (ACC) /American Heart Association (AHA) guidelines on dyslipidaemia, provide an important framework for the cardiovascular risk reduction strategy.
\end{abstract}

Objectives: To assess treatment gaps regarding statin therapy among patients without diabetes presenting to a medical clinic using standards set by the 2013 ACC/AHA guidelines.

Methodology: A descriptive cross-sectional study was conducted for 6 months from December 2016, at the outpatient medical clinic, Teaching Hospital Kandy. A sample of nondiabetic patients, between 40-75 years was included. Data such as patient demographics, ASCVDs, cardiac risk factors, blood pressure, lipid levels and statin history were gathered from patients and their clinic records. Statin requirement was assessed according to the guidelines.

Results: A total of 496 subjects were included. Mean age was 57.4 (SD 8.4) years and 66.5\% were females. $270(54.4 \%)$ patients were recognized to benefit from statin therapy, and the rest not to benefit from statin therapy. From 270 statin benefit patients, only $72(26.6 \%)$ were on the recommended dose of statin therapy and $70(25.9 \%)$ were not being treated with any statin. 144/226 (63.7\%) patients who were non statin benefit were taking a statin.

Conclusions: A majority of patients who required statin therapy were not on a statin or

\footnotetext{
1 Teaching Hospital, Kandy, Sri Lanka.

2 University of Kelaniya, Sri Lanka.
}

Corresponding author: Jayasinghe I $K$

E-mail: kumujay@gmail.com on the recommended dose, whereas a large number of patients who did not require a statin were on a statin. It is recommended to initiate statin therapy in appropriate dose after assessing total ASCVD risk.

\section{Introduction}

Non communicable diseases (NCDs) are the world's number one killer, in both rich and poor nations alike. Of the 56.9 million global deaths in 2016, 40.5 million, were due to NCDs' ${ }^{1}$. Over three quarters of NCDrelated deaths occurred in low and middle income countries $^{1,2}$. In Sri Lanka, 1 in 5 people die prematurely from NCDs ${ }^{3}$. According to Registrar General's data in 2001, NCDs accounted for $71 \%$ of total deaths in Sri Lanka ${ }^{4}$. The leading causes of NCD deaths were due to cardiovascular diseases ${ }^{1,2}$.

Atherosclerotic Cardiovascular Disease (ASCVD) is defined as acute coronary syndromes (ACSs) with a history of myocardial infarction (MI), stable or unstable angina, coronary or other arterial revascularization, ischaemic stroke, transient ischemic attack or peripheral arterial disease ${ }^{5,6}$. Dyslipidaemia, diabetes mellitus (DM), hypertension, smoking and family history of cardio vascular disease (CVD) are the major risk factors for ASCVD ${ }^{7}$. Control of such cardiovascular risk factors is crucial for the prevention of cardiovascular morbidity and mortality.

The guideline of the Third Adult Treatment Panel (ATP-III) of the National Cholesterol Education Program (NCEP) has been used globally since their release in 2002 in treatment of dyslipidaemia. According to NCEP ATP-III guideline, individuals at high risk for ASCVD with low density lipoprotein cholesterol (LDL-C) levels $\geq 100 \mathrm{mg} / \mathrm{dl}$ were candidates for statin therapy ${ }^{8}$. In 2011 European Society of Cardiology (ESC)/European Atherosclerosis Society (EAS) guidelines for the management of dyslipidaemia too had given target lipid levels with reference to LDL-C and other lipoproteins ${ }^{9}$. Both guidelines provided goals of target lipid levels in each risk category. 
In 2013, American College of Cardiology/American Heart Association (ACC/AHA) cholesterol guideline emphasized global cardiovascular (CV) risk reduction as opposed to targeting LDL-C levels. In this guideline, statins were grouped as high, moderate, and low intensity statin (Table 1).

Table 1. Classification of statins according to 2013 ACC/AHA guideline

\begin{tabular}{l|l|l}
\hline High-intensity Statin & Moderate-intensity Statin & Low-intensity Statin \\
\hline $\begin{array}{l}\text { Daily dose lowers LDL-C, on } \\
\text { average, by approximately } \geq 50 \%\end{array}$ & $\begin{array}{l}\text { Daily dose lowers LDL-C, } \\
\text { on average, by approximately } \\
30 \% \text { to }<50 \%\end{array}$ & $\begin{array}{l}\text { Daily dose lowers LDL-C, } \\
\text { on average, by }<30 \%\end{array}$ \\
\hline $\begin{array}{l}\text { Atorvastatin } 40-80 \mathrm{mg} \\
\text { Rosuvastatin } 20-40 \mathrm{mg}\end{array}$ & $\begin{array}{l}\text { Atorvastatin } 10-20 \mathrm{mg} \\
\text { Rosuvastatin } 5-10 \mathrm{mg} \\
\text { Simvastatin } 20-40 \mathrm{mg} \\
\text { Pravastatin } 40-80 \mathrm{mg}\end{array}$ & $\begin{array}{l}\text { Simvastatin } 10 \mathrm{mg} \\
\text { Pravastatin } 10-20 \mathrm{mg}\end{array}$ \\
\hline
\end{tabular}

According to this guideline, initiation of moderate or high intensity statin therapy based on the CV risk is a crucial factor in reducing ASCVD events. Statin therapy reduces ASCVD events across the spectrum of baseline LDL-C levels $\geq 70 \mathrm{mg} / \mathrm{dl}^{5,6}$. In the guideline four groups of individuals who have been shown to benefit from statin therapy were identified.

Four major statin benefit groups are listed below,

1) Those with clinical ASCVD

2) Those with primary elevation of LDL-C $\geq 190 \mathrm{mg} / \mathrm{dL}$

3) Patients with diabetes and aged 40 to 75 years with LDL-C 70 to $189 \mathrm{mg} / \mathrm{dL}$

4) Individuals aged 40 to 75 years with LDL-C 70 to $189 \mathrm{mg} / \mathrm{dl}$ and estimated 10 year ASCVD risk $\geq 7.5 \%$

For the primary prevention of ASCVD, the absolute 10-year risk of ASCVD should be used to guide the initiation of statin therapy. In individuals without clinical ASCVD or diabetes having LDL-C 70 to $189 \mathrm{mg} / \mathrm{dl} 10$-year ASCVD risk is calculated using the ASCVD risk calculator in order to decide the need for initiating statin therapy. 2013 ACC/AHA guidelines recommended the new Pooled Cohort Risk Assessment Equations developed by the Risk Assessment Work Group to estimate the 10-year ASCVD risk.

A large number of adult patients with cardiovascular risk factors attend the medical clinic at outpatient department, Teaching Hospital Kandy (THK). These risk factors include diabetes, hypertension, dyslipidemia, smoking and obesity. According to the
Ministry of Health Sri Lanka directive on screening for NCDs ${ }^{10,11}$, fasting blood sugar (FBS) and total cholesterol or lipid profile are arranged in all adults aged 35 years or more in this clinic. Patients are then assessed and treated accordingly. However, there is no clear idea on their current statin treatment regimens or need for statin treatment according to the cholesterol treatment guidelines.

\section{Objectives}

With the publication of 2013 ACC/AHA guidelines on cholesterol treatment, the clinicians in the medical clinic at outpatient department of THK had a concern as to whether patients were being treated for dyslipidaemia according to these recommendations. This clinical study was designed to review statin treatment of patients without diabetes in this medical clinic. The objectives included reviewing the ongoing statin therapy, assessing the need for changing the treatment according to new recommendations in the 2013 ACC/AHA guidelines and assessing the need for starting statin therapy for those who were not on statin in the selected study population.

\section{Methodology}

A descriptive cross-sectional study was conducted at the medical clinic at the outpatient department, THK. This medical facility treats a large number of patients including those with NCDs, from Monday to Saturday. Kandy hospital statistics showed a total of 6952 patients attended this clinic in 2015.

Non-diabetic patients between 40 to 75 years, with clinical ASCVDs and /or cardiovascular risk 
factors other than diabetes, were included in the study. The eligible patients were identified from the clinic records. The study was conducted over 6 months starting from mid-December 2016. Written informed consent was obtained from the participants. All the investigators were educated about this study in detail with power point presentations on the $2013 \mathrm{ACC} / \mathrm{AHA}$ guidelines, on research methodology, ethical considerations and how to clarify and solve issues during the study period. These power point presentations were saved in each investigator's personal computer, tablet or smart phone for further reference.

All participants were interviewed and the clinic records were reviewed to collect information using a standard questionnaire by clinical investigators. Individual data belonging to each patient was saved with a unique identification number in the electronic form using Microsoft Office Excel. Their contact details too were recorded for future references.

Collected data included: demographic data (age and gender), presence or absence of clinical ASCVD (Ischaemic Heart Disease (IHD), arterial revascularization (CABG/PCI), Peripheral Vascular Diseases (PVD), ischaemic stroke /TIA), BP value and history of cardiovascular risk factors such as hypertension, dyslipidemia, smoking, and family history of cardiovascular disease. Pre-treatment lipid profile details, details regarding statin therapy (name, dose, contraindications, side effects such as allergy, myopathy or elevated liver serum transaminases (SGPT)) were also collected.

Those without lipid details, not on statin therapy due to side effects or contraindications and having secondary causes of dyslipidemia were excluded from the study. The patients included in the study were categorized into four clinical groups as follows.

1. Patients with clinical ASCVD

2. Patients without $A S C V D$ having $L D L-C \geq 190 \mathrm{mg} / \mathrm{dl}$

3. Patients without ASCVD having LDL-C $70-189 \mathrm{mg} /$ $\mathrm{dl}$ and 10 year ASCVD risk $\geq 7.5 \%$

4. Patients without ASCVD having LDL-C 70-189 $\mathrm{mg} / \mathrm{dl}$ and 10 year ASCVD risk $<7.5 \%$ or LDL-C $<70 \mathrm{mg} / \mathrm{dl}$.

According to 2013 ACC/AHA guidelines; first two groups require a high intensity statin therapy. The third group requires a moderate intensity statin therapy whereas statin therapy is not indicated for the fourth group. In third and fourth groups 10-year ASCVD risk was calculated using the ASCVD risk calculator downloaded from AHA/ACC web site to smart mobile phones of the investigators. (http://tools.acc.org/ ASCVD-Risk-Estimator/)

\section{Ethical considerations}

Ethical approval was taken from the institutional Ethical Review Committee, University of Peradeniya. Informed written consent was obtained from the participants.

\section{Data analysis}

All statistical analysis was conducted using SPSS version 21.0 software. Nominal variables were presented as the number of cases and percentages, and continuous variables were presented as means \pm standard deviations. Statin treatment regimens of the study groups were compared with the treatment recommendations of 2013 ACC/AHA guidelines.

\section{Results}

\section{Demographic and baseline values}

The study group consisted of 496 patients after excluding 2 patients. The majority $330(66.5 \%)$ were females. The mean age was 57.4 (SD 8.4) years. Among the participants, the cardiovascular risk factors detected were: dyslipidaemia in $80.2 \%$, hypertension in $78.4 \%$, family history of CVD in $21.6 \%$ and smoking in $10.7 \%$. There were 55 patients with established ASCVD with 51 having ischaemic heart disease (IHD) and 6 patients having ischaemic stroke or transient ischemic attack (TIA). 2 patients had both IHD and Stroke/ TIA.

Total of $270(54.4 \%)$ out of 496 subjects were identified to benefit from statin therapy. There were 55 (11.1\%) patients in Group 1 who had clinical ASCVD and 76 (15.3\%) patients in Group 2 who had LDL $\geq 190$ $\mathrm{mg} / \mathrm{dl}$, requiring high intensity statin therapy. $139(28 \%)$ patients were in Group 3 with 10-year ASCVD risk ?7.5\% requiring moderate intensity statin therapy. 226 individuals (45.6\%) were in Group 4 with 10-year ASCVD risk $<7.5 \%$ and not indicated to benefit from statin therapy (Table 2).

\section{Statin use in statin benefit groups}

Out of the total 270 patients who were eligible to have statin therapy (patients in Group 1,2 and 3) only $72(26.7 \%)$ were on an adequate dose of statin as recommended by 2013 ACC/AHA cholesterol guidelines. Among the 55 patients with clinical ASCVD (Group 1) only two patients were on high intensity statin. The majority, 51 patients were on inadequate doses and two patients were not on statin therapy with no valid reason. 
Table 2. Number of patients with statin requirement in each clinical group

\begin{tabular}{|c|c|c|c|}
\hline Group & Number & Percentage & Statin Requirement \\
\hline 1 - Patients with ASCVD & 55 & $11.1 \%$ & High intensity statin (Atorvastatin $40-80 \mathrm{mg}$ ) \\
\hline 2 - LDL $\geq 190 \mathrm{mg} / \mathrm{dl}$ & 76 & $15.3 \%$ & \\
\hline $\begin{array}{l}3 \text { - 10-year ASCVD risk } 7.5 \% \\
\text { and LDL } 70-189 \mathrm{mg} / \mathrm{dl}\end{array}$ & 139 & $28.0 \%$ & Moderate intensity statin (Atorvastatin $10-20 \mathrm{mg}$ ) \\
\hline $\begin{array}{l}4-10 \text {-year ASCVD risk } \\
<7.5 \% \text { or LDL }<70 \mathrm{mg} / \mathrm{dl}\end{array}$ & 226 & $45.6 \%$ & Statin is not required \\
\hline
\end{tabular}

In group 2 with $L D L \geq 190 \mathrm{mg} / \mathrm{dll}$, out of 76 patients none were on recommended high intensity statin; 62 were on an inadequate dose and 14 patients were not on a statin at all. From total 139 patients who belong to Group 3 with 10-year ASCVD risk $\geq 7.5 \%, 70$ were receiving the required moderate intensity statin therapy and 15 patients were treated with an inadequate dose of statin. 54 patients were not on a statin in this group as well. (Figure 1). This study identified 70 patients who required statin therapy but not taking a statin at the time of the study. There were total of 226 patients in Group 4 who did not require statin therapy. However, the majority, $144(63.7 \%)$ patients were taking a statin in this group.

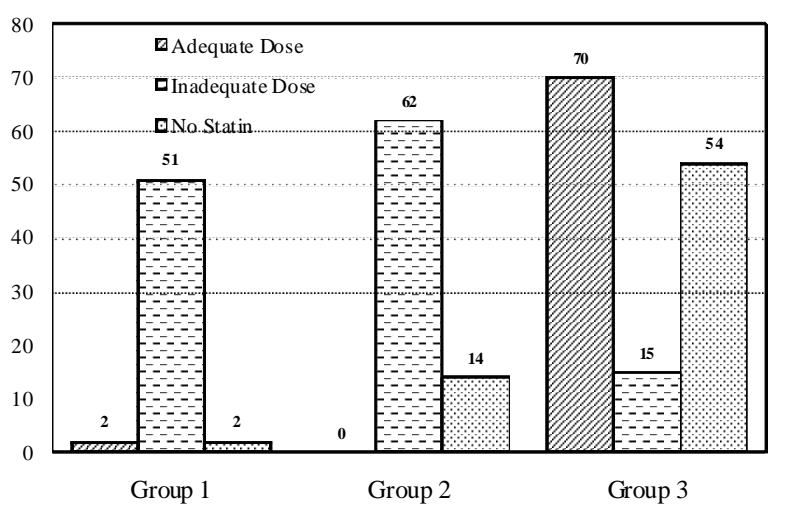

Figure 1. Statin therapy in statin benefit groups.

\section{Discussion}

This study was conducted to assess statin use according to 2013 ACC/AHA cholesterol guidelines, in non-diabetic patients with cardiovascular risk factors and/or established cardiovascular diseases in one of the medical clinics in Central Province of Sri Lanka.
According to the results, the majority $(129 / 131)$ in the high-risk groups in this population (Group 1 with clinical ASCVD and Group 2 with LDL $\geq 190 \mathrm{mg} / \mathrm{dl}$ ) were not on statin therapy or treated with sub-optimal doses. This would increase possible future CVD events. Out of 139 patients who required moderate intensity statin (i.e. Group 3), approximately half $(n=70)$ were treated with the recommended dose. In this group a significant proportion, $38.8 \%(n=54)$ was not treated though they required statin therapy according to the guidelines.

This study detected 70 new patients who would benefit from statin therapy. Among the 226 who were not eligible for statin treatment according to the guideline, more than $50 \%(144 / 226)$ were on a statin.

A study done in the USA to assess statin use according to 2013 ACC/AHA cholesterol guidelines in 4536 primary care patients aged 40 to 75 years with at least one outpatient visit showed that $32 \%$ would need to be started on a statin, $12 \%$ required an increased dose, and $6 \%$ could stop statins ${ }^{12}$. In a similar study on 3203 non-diabetic patients aged 40 to 75 years, 2008 were not on a statin and among them, there were $466(23.2 \%)$ who fit to criteria for initiation of moderate or high intensity statin therapy ${ }^{13}$.

The largest impact on the healthcare system with application of the 2013 ACC/AHA cholesterol guidelines is the recommendation of statins for primary prevention in individuals with a 10-year ASCVD risk of $7.5 \%$ or higher. The previous recommendations considered a substantially higher threshold for 10-year risk of fatal and non-fatal coronary heart disease. A study done in the USA, based on this guideline, showed the change in usage of statin. It estimated a $25 \%$ increase in statin use, increasing from $3,909,407(27.7 \%)$ patients to $4,892,668(34.7 \%)$ patients, for the projected three year period from 2014. These projected changes would increase the overall number of statin prescriptions by 
$25 \%$ and would decrease the number of non-statin cholesterol lowering medication prescriptions by $68 \%$ during the projected period ${ }^{14}$. The same projections in usage of statin could be expected in Sri Lanka with application of this guideline.

As more people are treated with moderate to high intensity statins, there would be more individuals who would develop side effects and statin intolerance. Although statins are generally well tolerated and present a good safety profile, adverse effects such as muscle toxicity and effects on liver enzymes may occur in some patients ${ }^{15}$. According to a review approximately $15 \%$ are intolerant to statin ${ }^{16}$. Statin adverse effects have been well documented in some other reviews, with muscle related toxicity receiving the greatest attention ${ }^{17,18}$. These reviews provide an update on the safety of statins incorporating the more recent findings from meta analyses which have shown that intensive statin therapy is associated with a slightly increased risk of the development of diabetes ${ }^{19}$.

There were no randomised control trials of Sri Lankan population to decide the goal of ideal statin therapy. In the absence of a specific goal for LDL-C levels in Sri Lankan population, the clinicians in Sri Lanka have to rely on guidelines meant for other populations in the world. As those with Asian ancestry are more sensitive to the effects of statins, whether our patients here are really in need of these high doses recommended by 2013 ACC/AHA guideline is another concern that needs to be addressed in the future.

It is a well established fact that statins have multiple effects other than lipid lowering such as antiinflammatory effects, antioxidant effects, antiproliferative effects, plaque stability and prevention of platelet aggregation. These multiple lipid independent effects of statins are termed statin pleiotropy [20]. According to a review, pleiotropic effects of statins are an important consideration in statin therapy irrespective of the baseline LDL-C levels, as the benefits of statin therapy exceed those predicted by cholesterol reduction alone. This is in favour of the $2013 \mathrm{ACC} /$ AHA dyslipidaemia management. Therefore, Sri Lankan clinicians must take the pleiotropic effect benefits into account when planning the treatment of aggressive atherosclerotic disease ${ }^{21}$. Until data from prospective studies on Sri Lankan population are available, currently available clinical evidences and guidance should be used with caution.

\section{Limitations}

Risk estimation calculations using an American risk calculator may not be accurate for our sample. A Sri Lankan community based cross sectional study done in an urban population to assess CVD risk, using three different risk assessment charts showed three different risk estimates ${ }^{22}$. Other limitations were that the study was limited to the age range of 40 to 75 years and life style changes and other lipid lowering medications other than statins were not addressed in this study group.

\section{Conclusion and recommendations}

A majority of patients who need high intensity statin therapy were not on statin therapy at all or did not receive the recommended dose, whereas a large number of patients who did not belong to a statin benefit group, were on statin therapy. It is recommended to initiate statin therapy after assessing total ASCVD risk rather than concentrating on lipid levels alone. Development of a national guideline for management of dyslipidaemia is highly recommended.

\section{Acknowledgement}

Mr I M Gunawardana - Former Deputy Director, Faculty of Agriculture, University of Peradeniya for creating patient information and consent forms.

\section{Conflicts of interests}

There were no conflicts of interest.

This study was carried out at the VP/OPD Clinic, Teaching Hospital, Kandy.

All authors consented to the publishing of this paper in the journal of CCP.

\section{References}

1. World Health Organization. NCD mortality and morbidity https:/ /www.who.int/gho/ncd/mortality_morbidity/en/

2. NCD Alliance. The global epidemic. https://ncdalliance.org/ the-global-epidemic

3. World Health Organization. Sri Lanka Noncommunicable diseases: Sri Lanka country profile. https://www.who.int/ beat-ncds/countries/sri-lanka/en/

4. Registrar General's Data Sri Lanka- 2001.

5. Stone NJ, Robinson JG, Lichtenstein AH, et al. 2013 ACC/ AHA guideline on the treatment of blood cholesterol to reduce atherosclerotic cardiovascular risk in adults: Circulation 2014; 129: S1-S45

6. Goff DC, Lloyd-Jones DM, Bennett G, et al. 2013 ACC/AHA guideline on the assessment of cardiovascular risk: a report of the American College of Cardiology/American Heart Association Task Force on Practice Guidelines. Circulation 2014; 129: S49-S73 
7. Brian Boudin F . Risk Factors for Coronary Artery Disease. Medscape, e medicine. ttps://emedicine.medscape.com/ article/164163-overview

8. Grundy SM, Cleeman JI, Merz CN, et al. Coordinating Committee of the National Cholesterol Education Program. Implications of recent clinical trials for the National Cholesterol Education Program Adult Treatment Panel III Guidelines. Journal of American College of Cardiology 2004; 44: 720-32.

9. Reiner Z, Catapano AL, Graham I, et al. ESC/ EAS Guidelines for the management of dyslipidaemias. European Heart Journal 2011; 32: 1769-8.

10. The national policy and strategic framework for prevention and control of chronic non-communicable diseases, Ministry of Health Sri Lanka -2009.

11. Ministry of Health Sri Lanka Circular 41/2011.

12. Schoen MW, Salas J, Scherrer JF, Buckhold FR. Cholesterol treatment and changes in guidelines in an academic medical practice. American Journal of Medicine 2015; 128(4):403-9

13. Koenigsfeld C, Sayler M, Smith HL,et al. Retrospective Evaluation of ASCVD Risk and Statin Therapy Need in Nondiabetic Patients Based on the 2013 ACC/AHA Cholesterol Guidelines. Journal of Pharmacy Practice 2016: 30(3): 300-5.

14. Tran JN, Caglar T, StockI KM, Lew HC, Solow BK, Chan PS.
Impact of the New ACC/AHA Guidelines on the Treatment of High Blood Cholesterol in a Managed Care Setting. American Health and Drug Benefits 2014 ; 7(8): 430-43.

15. Miao Hu. Safety of statins: an update. Therapeutic Advances in Srug Safety 2012; 3(3): 133-44.

16. Kones R, Rumana U, Current Treatment of Dyslipidaemia: A New Paradigm for Statin Drug Use and the Need for Additional Therapies. Drugs 2015 ; 75(11): 1187-99.

17. Armitage J. The safety of statins in clinical practice. Lancet 2007; 370(9601): 1781-90.

18. Brown WV. Safety of statins. Current Opinion in Lipidology. 2008; 19(6): 558-62.

19. Sattar $N$ et al. Statins and risk of incident diabetes: a collaborative meta-analysis of randomised statin trials. Lancet 2010; 375(9716): 735-42.

20. Narasaraju K, Jay S, Ananthraman R, Hardik V. Pleotrophic effects of statins. Indian Journal of Endocrinology and Metabolism 2015; 19(5): 554-62.

21. Ruvan Al, Ekanayaka. The changing paradigm of dyslipidaemia management. Universa Medicina. 2017; 36: 1-3.

22. Ranawaka, UK, Wijekoon CN, Pathmeswaran A, et al. Risk estimates of cardiovascular diseases in a Sri Lankan community. Ceylon Medical Journal 2016; 61(1): 11-7. 